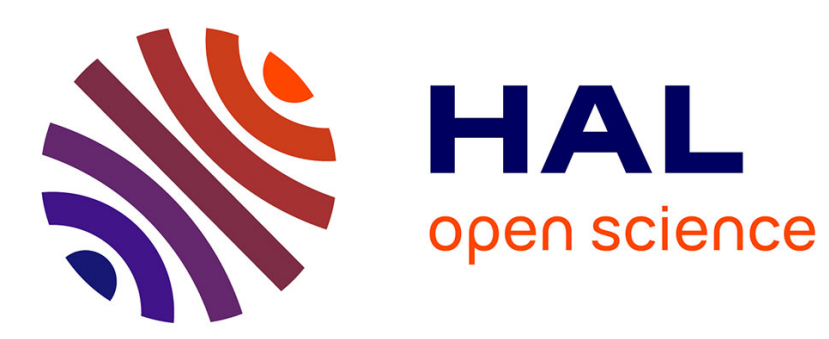

\title{
Solving the Quadratic Assignment Problem with Cooperative Parallel Extremal Optimization
}

\author{
Danny Munera, Daniel Diaz, Salvador Abreu
}

\section{To cite this version:}

Danny Munera, Daniel Diaz, Salvador Abreu. Solving the Quadratic Assignment Problem with Cooperative Parallel Extremal Optimization. 16th European Conference on Evolutionary Computation in Combinatorial Optimization, EvoCOP 2016, Mar 2016, Porto, Portugal. 10.1007/978-3-319-306988_17. hal-01332524

\section{HAL Id: hal-01332524 https://hal.science/hal-01332524}

Submitted on 16 Jun 2016

HAL is a multi-disciplinary open access archive for the deposit and dissemination of scientific research documents, whether they are published or not. The documents may come from teaching and research institutions in France or abroad, or from public or private research centers.
L'archive ouverte pluridisciplinaire HAL, est destinée au dépôt et à la diffusion de documents scientifiques de niveau recherche, publiés ou non, émanant des établissements d'enseignement et de recherche français ou étrangers, des laboratoires publics ou privés. 


\title{
Solving the Quadratic Assignment Problem with Cooperative Parallel Extremal Optimization
}

\author{
Danny Munera ${ }^{1}$, Daniel Diaz ${ }^{1}$ and Salvador Abreu ${ }^{2,1}$ \\ 1 University of Paris 1-Sorbonne/CRI, France \\ 2 Universidade de Évora/LISP, Portugal
}

\begin{abstract}
Several real-life applications can be stated in terms of the Quadratic Assignment Problem. Finding an optimal assignment is computationally very difficult, for many useful instances. We address this problem using a local search technique, based on Extremal Optimization and present experimental evidence that this approach is competitive. Moreover, cooperative parallel versions of our solver improve performance so much that large and hard instances can be solved quickly.
\end{abstract}

Keywords: QAP, extremal optimization, heuristics, parallelism, cooperation

\section{Introduction}

The Quadratic Assignment Problem (QAP) was introduced in 1957 by Koopmans and Beckmann 1 as a model of a facilities location problem. This problem consists in assigning a set of $n$ facilities to a set of $n$ specific locations minimizing the cost associated with the flows of items among facilities and the distance between them. This combinatorial optimization problem has many other real-life applications: scheduling, electronic chipset layout and wiring, process communications, turbine runner balancing, data center network topology, to cite but a few 223. Unfortunately this problem is known to be NP-hard and finding efficient algorithms to solve it has attracted a lot of research in recent years.

Exact (or complete) methods like dynamic programming, cutting plane techniques and branch \& bound procedures have been successfully applied to mediumsize QAP instances but cannot solve larger instances (e.g. when $n>20$ ). To tackle these problems, one must resort to incomplete methods which are designed to quickly provide good, albeit sub-optimal, solutions. This is the case of approximation algorithms, i.e. algorithms running in polynomial time yet able to guarantee solutions within a constant factor of the optimum. Unfortunately, it is known that there is no $\epsilon$-approximation algorithm for the QAP [4. Another class of incomplete methods is provided by meta-heuristics. Since 1990 several meta-heuristics have been successfully applied to the QAP: tabu search, simulated annealing, genetic algorithms, GRASP, ant-colonies [3. The current trend is to specialize existing heuristics, to compose different meta-heuristics (hybrid procedures) and to use parallelism. 
In this paper we propose EO-QAP: an Extremal Optimization (EO) procedure for QAP. EO is a nature-inspired general-purpose meta-heuristics to solve combinatorial optimization problems [5]. This local search procedure, a priori, has several advantages: it is easy to implement, it does not get confounded by local minima and takes only one adjustable parameter. We experimentally demonstrate that EO-QAP performs well on the set of QAPLIB benchmark instances. It is, however, known that it is difficult with EO to have fine control on the trade-off between search intensification and diversification: some strategies have been proposed to overcome this limitation [6], but they entail a more complex tuning process. In this paper we put forth two other approaches which contribute to a more effective handling of QAP using EO: firstly, we propose a simple extension to the original EO which allows the user to have more control over the stochastic behavior of the algorithm. Secondly, we propose to use cooperative parallelism to promote more intensification and/or diversification. Our implementation uses a parallel framework [7] written in X10 [89]. We show that the cooperative parallel version behaves very well on the hardest instances.

The rest of the paper is organized as follows. Section 2 discusses QAP and provides the necessary background. Section 3 presents our EO algorithm for QAP and proposes an extension to the original EO. Several experimental results are laid out and discussed in section 4 and we conclude in Section 5.

\section{Background}

Before introducing the main object of this paper, we need to recall some background topics: the Quadratic Assignment Problem (QAP), Extremal Optimization (EO) and Cooperative Parallel Local Search (CPLS).

\subsection{QAP}

Since its introduction in 1957, QAP has been widely studied and several surveys are available $10[2] 113$.

A QAP problem of size $n$ consists of two $n \times n$ matrices $\left(a_{i j}\right)$ and $\left(b_{i j}\right)$. Let $\Pi(n)$ be the set of all permutations of $\{1,2, \ldots n\}$, the goal of QAP is to find a permutation $\pi \in \Pi(n)$ which minimizes the following objective function:

$$
F(\pi)=\sum_{i=1}^{n} \sum_{j=1}^{n} a_{i j} \cdot b_{\pi_{i} \pi_{j}}
$$

For instance, in facility location problems, the $a$ matrix represents interfacility flows and $b$ encodes the inter-location distances. In that context, both matrices are generally symmetric: $\forall_{i, j} a_{i j}=a_{j i}$ and $b_{i j}=b_{j i}$. However, in other settings the matrices can become asymmetric. Indeed, QAP can be used to model scheduling, chip placement and wiring on a circuit board, to design typewriter keyboards, for process communications, for turbine runner balancing among many other applications 212]. 
The computational difficulty of QAP stems form the fact that the objective function contains products of variables (hence the term quadratic) and in the fact that the theoretical search space of an instance of size $n$ is the set of all permutations $\Pi(n)$ whose cardinality is $n$ !. In 1976, Sahni and Gonzalez proved that QAP is NP-hard 4] (the famous traveling salesman problem can be formulated as a QAP). Moreover, the same authors proved that there is no $\epsilon$-approximation algorithm for QAP (unless $\mathrm{P}=\mathrm{NP}$ ). In practice QAP is one of the most difficult combinatorial optimization problems with many real-life applications.

QAP can be (optimally) solved with exact methods like dynamic programming, cutting plane techniques and branch \& bound algorithms (together with efficient lower bound methods). Constraint Programming does not work well on QAP and, surprisingly, SAT solvers have not been extensively used for QAP. However, general problems of medium size (e.g. $n>20$ ) are out of reach for these methods (even if some particular larger instances can be solved). It is thus natural to use heuristics to solve QAP. In the last decades several meta-heuristics were successfully applied to QAP: tabu search, simulated annealing, genetic algorithms, GRASP, ant-colonies [13. In this paper we propose to use Extremal Optimization to attack QAP problems.

\section{$2.2 \quad$ Extremal Optimization}

In 1999, Boettcher and Percus proposed the Extremal Optimization (EO) procedure [5]14]15] as a meta-heuristics to solve combinatorial optimization problems. EO is inspired by self-organizing processes often found in nature. It based on the concept of Self-Organized Criticality (SOC) initially proposed by Bak [16]17] and in particular by the Bak-Sneppen model of SOC [18. In this model of biological evolution, species have a fitness $\in[0,1]$ (0 representing the worst degree of adaptation). At each iteration, the species with the worst fitness value is updated, i.e. its fitness is replaced by a new random value. This change also affects all other species connected to this "culprit" element and their fitness value also gets updated. This results in an extremal process which progressively eliminates the least fit species (or forces them to mutate). Repeating this process eventually leads to a state where all species have a good fitness value, i.e. a SOC. EO follows this line: it inspects the current configuration (assignment of variables), selects the worst variable (the one having the lowest fitness) and replaces its value by a random value. However, always selecting the worst variable can lead to a deterministic behavior and the algorithm can stay blocked in a local minimum. To avoid this, the authors propose an extended algorithm which first ranks the variables in increasing order of fitness (the worst variable has thus a rank $k=1$ ) and then resorts to a Probability Distribution Function (PDF) over the ranks $k$ in order to introduce uncertainty in the search process:

$$
P(k)=k^{-\tau} \quad(1 \leq k \leq n)
$$

This power-law probability distribution takes a single parameter $\tau$ which is problem-dependent. Depending on the value of $\tau$, EO provides a wide variety of 
search strategies from pure random walk $(\tau=0)$ to deterministic (greedy) search $(\tau \rightarrow \infty)$. With an adequate value for $\tau$, EO cannot be trapped in local minima since any variable is susceptible to mutate (even if the worst are privileged). This parameter can be tuned by the user. Moreover, the original paper proposes a default value depending on $n: \tau=1+\frac{1}{\ln (n)}$.

EO displays several a priori advantages: it is a simple meta-heuristic (it can be easily programmed), it is controlled by only one free parameter (a fine tuning of several parameters becomes quickly tedious) and it does not need to be aware about local minima. Nevertheless, EO has been successfully applied to large-scale optimization problems like graph bi-partitioning, graph coloring, Spin Glasses or the traveling salesman problem [14. Boettcher and Percus point out, however, that depending on the problem, "a drawback of the EO method is that a general definition of fitness for the individual variables may prove ambiguous or even impossible" [19. To overcome this, De Sousa and Ramos proposed an extension called Generalized Extremal Optimization 20|21. Zhou and al. proposed a variant called Continuous Extremal Optimization to deal with continuous optimization problems [22]. It has been also argued that one main issue with EO is that is does not provide a fine control of the intensification. Randall and Lewis propose some intensification strategies to improve EO [6]. We present two alternatives to overcome this limitation: we propose a simple extension to improve the stochastic capabilities of EO and we show how cooperative parallelism can help to achieve intensification or diversification through communications.

\subsection{Cooperative Parallel Local Search}

Parallel local search methods have been proposed in the past 23|24|25]. In this article we are interested in multi-walk methods (also called multi-start) which consist in a concurrent exploration of the search space, either independently or cooperatively via communication between processes. The Independent MultiWalks method (IW) [26] is the easiest to implement since the solver instances do not communicate with each other. However, the resulting gain tends to flatten when scaling over a hundred of processors 27, and can be improved upon. In the Cooperative Multi-Walks (CW) method [28], the solver instances exchange information (through communication), hoping to hasten the search process. However, implementing an efficient cooperative method is a very complex task: several choices have to be made about the communication which influence each other and which are problem-dependent [28].

We build on the framework for Cooperative Parallel Local Search (CPLS) proposed in [2977. This framework, available as an open source library in the X10 programming language, allows the programmer to tune the search process through an extensive set of parameters which, in the present version, statically condition the execution. CPLS augments the IW strategy with a tunable communication mechanism, which allows for the cooperation between the multiple instances to seek either an intensification or diversification strategy for the search. At present, the tuning process is done manually: we have not yet experimented with parameter self-adaptation (still an experimental feature). 
The basic component of CPLS is the explorer node which consists in a local search solver instance. The point is to use all the available processing units by mapping each explorer node to a physical core. Explorer nodes are grouped into teams, of size NPT (see Figure 11). This parameter is directly related to the trade-off between intensification and diversification. NPT can take values from 1 to the maximum number of nodes (frequently linked to maximum number of available cores in the execution). When $N P T$ is equal to 1 , the framework coincides with the IW strategy, it is expected that each 1-node team be working on a different region of the search space, without seek parallel intensification. When NPT is equal to the maximum number of nodes (creating only 1 team in the execution), the framework has the maximum level of parallel intensification, but it is not possible to enable parallel diversification between teams.
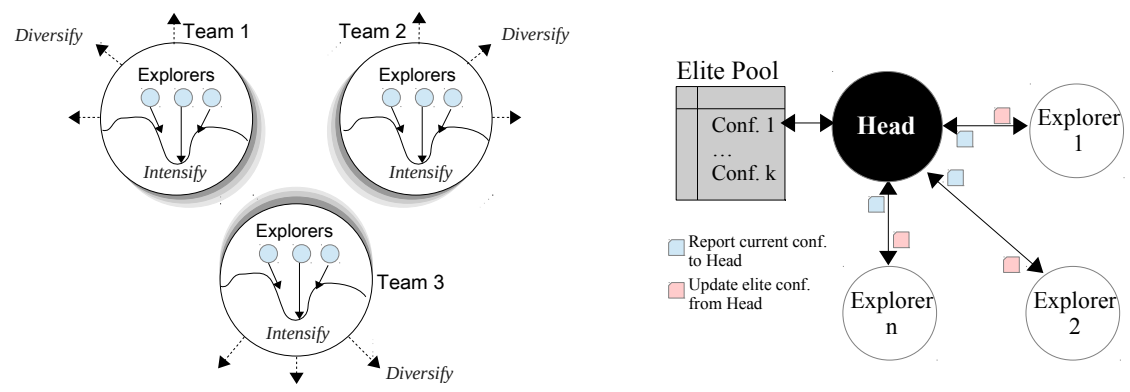

Fig. 1: CPLS framework structure

Each team seeks to intensify the search in the most promising neighborhood found by any of its members. The parameters which guide the intensification are the Report Interval $(R)$ and Update Interval $(U)$ : every $R$ iterations, each explorer node sends its current configuration and the associated cost to its head node. The head node is the team member which collects and processes this information, retaining the best configurations in an Elite Pool $(E P)$ whose size $|E P|$ is parametric. Every $U$ iterations, explorer nodes randomly retrieve a configuration from the $E P$, in the head node. An explorer node may adopt the configuration from the $E P$, if it is "better" than its own current configuration with a probability $p$ Adopt. Simultaneously, the teams implement a mechanism to cooperatively diversify the search, i.e. they try to extend the search to different regions of the search space.

Typically, each problem benefits from intensification and diversification on some level. Therefore, the tuning process of the CPLS parameters seeks to provide the appropriate balance between the use of the intensification and diversification mechanisms, in hope of reaching better performance than the noncooperative parallel solvers (e.g. Independent Multi-Walks). A detailed description of this framework may be found in [7]. 


\section{EO-QAP: an EO Procedure for QAP}

\subsection{General Procedure}

Our EO-QAP algorithm starts from a random permutation $\pi \in \Pi(n)$, with an associated cost given by $F(\pi)$. To ensure we only consider proper permutations, we only perform swap operations on pairs of elements from any given one: this is how value assignment is classically implemented in permutation problems, thereby eschewing the costly explicitly encoding of an all-different constraint. We define the permutation resulting from swapping $\pi_{i}$ and $\pi_{j}$ :

$$
\pi_{i \leftrightarrow j}=\mu \mid \mu_{i}=\pi_{j}, \mu_{j}=\pi_{i}, \mu_{k}=\pi_{k} \quad \forall k \notin\{i, j\}
$$

The neighborhood of $\pi_{i}$ is the set $N\left(\pi_{i}\right)$ of the permutations obtained from $\pi$ by swapping $\pi_{i}$ with any another value. By extension, the neighborhood of $\pi$ is the set $N(\pi)$ of all permutations obtained by swapping any two values:

$$
\begin{gathered}
N\left(\pi_{i}\right)=\left\{\pi_{i \leftrightarrow j} \mid 1 \leq j \leq n, j \neq i\right\} \\
N(\pi)=\bigcup_{i=1}^{n} N\left(\pi_{i}\right)
\end{gathered}
$$

Most local search procedures (in particular hill climbing) would select, among all elements of $N(\pi)$, the best neighbor $\mu$, i.e. the one minimizing the next global cost function. By doing so, they have to deal with the problem of being trapped in a local minimum. Instead, EO defines a fitness value $\lambda_{i}$ for each value $\pi_{i}$, with the understanding that a value with a low fitness is more likely to mutate, i.e. to get swapped. We define the fitness value $\lambda_{i}$ as the best possible improvement of the cost $F$ when moving to a $\pi_{i}$ 's neighbor.

$$
\lambda_{i}=\min _{\mu \in N\left(\pi_{i}\right)} F(\mu)-F(\pi)
$$

A negative $\lambda$ thus represents an improvement of the cost. At each iteration, the $n$ fitness values are evaluated and ranked, with rank $k=1$ for the worst fitness. EO-QAP will thus favor the mutation of a value which improves (decreases) the objective function. The value to mutate is chosen stochastically from a probability distribution over the rank order. This comes down to pick a value at rank $k(1 \leq k \leq n)$ with a probability $P(k)=k^{-\tau}$. Let $\pi_{r}$ be the value measured by the $k^{t h}$ fitness value, then $\pi_{r}$ will be deemed the "culprit" and be forced to mutate. For this we need to choose a target value $\pi_{s}$ for the swap. Several possibilities exist: one is to choose $\pi_{s}$ randomly. Another, as in the original EO article, is to pick a random value using the same probability distribution. We propose a third possibility applying the min-conflict heuristic [30] : select the best possible value, that is, the value which minimizes the objective function of the next configuration. The algorithm then swaps $\pi_{r}$ and $\pi_{s}$ (thus moving to a neighbor) and iterates with this new configuration. The process stops when a some condition is reached (e.g. a time limit or a given cost is reached). The best solution found so far is then returned (see Algorithm 1 . 


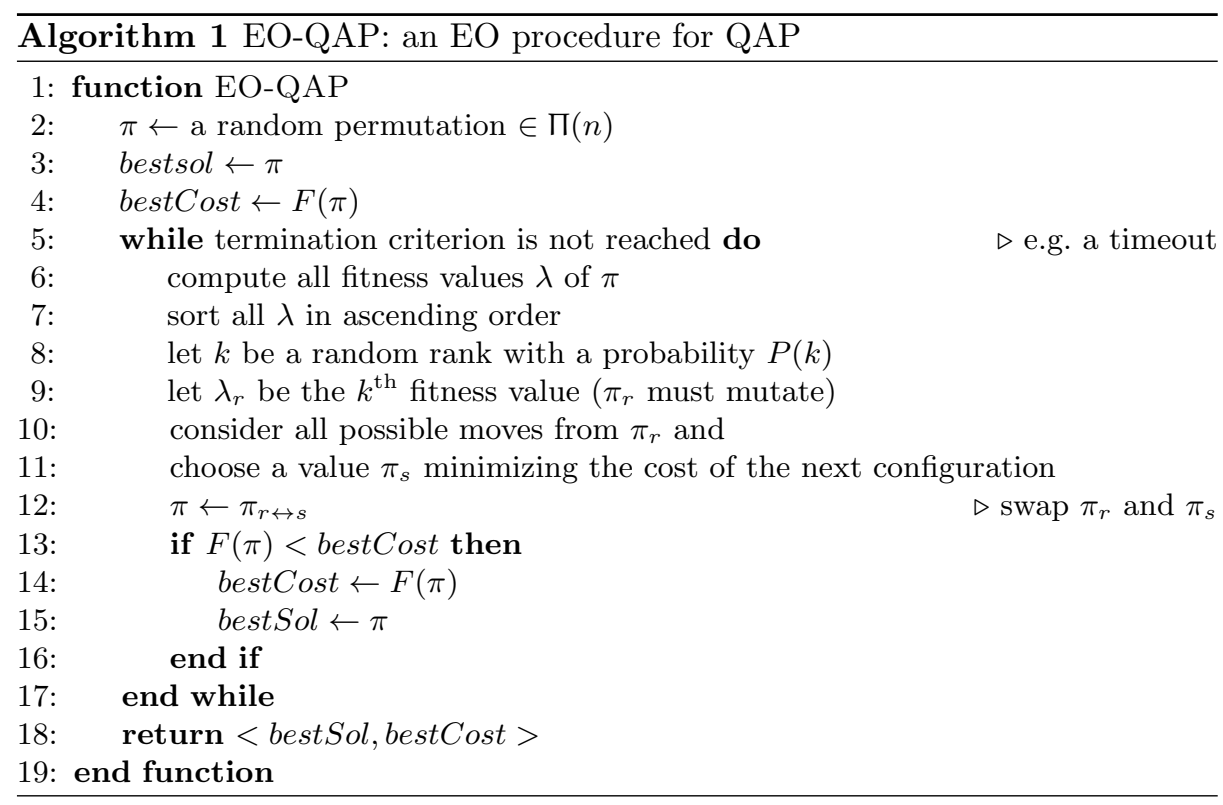

Implementation Commentary and Complexity Analysis. A permutation $\pi$ can be encoded by an array of $n$ integers sol [] (with sol [i] $=\pi_{i}$ ). Line 6 computes the fitness $\lambda_{i}$ for each value $\pi_{i}$. Thanks to Taillard and his famous Robust Taboo Search (RoTS), we know how to compute the evolution of the global cost after a swap incrementally, instead of recomputing it each time from scratch - see equations (1) and (2) which define $\Delta(\mu, .,$.$) in 31. This results$ in an evaluation of all $\lambda$ in $O\left(n^{2}\right)$, while a naïve algorithm is in $O\left(n^{3}\right)$. The simplest data structure to manage $\lambda$ is just an array fit [] whose elements are pairs of the form $<$ index,lambda $>$. Initially we have $f i t[i]=\left\langle i, \lambda_{i}\right\rangle$. Line 7 sorts the fit [] array on the second field $(\lambda)$, in ascending order, this can be done in $O\left(n \log _{2}(n)\right)$. Line 8 picks a value at position $k$ with a probability $P(k)$. Since the PDF and $\tau$ are constant along the execution of the algorithm, it is more efficient to pre-compute the $n$ samples of $P(k),(1 \leq k \leq n)$ and store then in an array prob []. To pick a random $k$, we can use a roulette-wheel selection on prob [] in $O(n)$ theoretically (but closer to $O(1)$ in practice due to the PDF). It is also possible to use a a binary search in $O\left(\log _{2}(n)\right)$ storing the cumulative PDF (prob $[\mathrm{k}]=\sum_{i=1}^{k} P(i)$ ). Line 9. the variable to mutate is given by sol [fit $[\mathrm{k}]$. index]. Line 10 selects the other variable for the swap, as per the min-conflict heuristic; this is done in $O(n)$. However, as this variable had already been found when computing $\lambda$ (Line 6), one just has to record it. To this end, the elements of the fit [] array are refactored as $<$ index,lambda,index $2>$, where index 2 contains the index of the variable which minimizes the cost. The second variable for the swap is then simply given by sol [fit $[\mathrm{k}]$.index2]. The overall complexity of each iteration (i.e. of the main loop body) is thus $O\left(n^{2}\right)$. 


\subsection{Extending Extremal Optimization}

Because it relies on just one parameter, EO is comparatively very simple to use (tuning many local search parameters can become very laborious). As we show in section 4 4 the results of EO-QAP are very good on many instances of QAPLIB. However, some harder instances need a fine control of the tradeoff between intensification and diversification. EO handles these two strategies with the same tool: the probability distribution $P(k)=k^{-\tau}$. Depending on the returned value, either the current path continues to be improved (with a high probability) or, in the extreme case, completely abandoned (with a low probability). Every variable has a non-zero chance of being selected and EO is not affected by local extrema. The choice of the probability distribution $P$ has thus a great impact, also determined by its parameter $\tau$, which must be selected by the user. Tuning $\tau$ for some hard problems (e.g. tai40a) turned out to be difficult. We therefore decided to extend $\mathrm{EO}$ so as to accept different probability distribution functions (PDF). The user can thus choose the most appropriate PDF. For simplicity, all proposed PDFs take a single input parameter $\tau$, the other parameters (if any) being either constant or functionally dependent on $\tau$. We now discuss a few interesting PDFs, and how they are influenced by the single $\tau$ parameter:

\begin{tabular}{lll} 
PDF definition & Usage in EO & Name \\
\hline $\operatorname{Power}(x, \tau)=x^{-\tau}$ & $P(k)=\operatorname{Power}(k, \tau)$ & power law \\
$\operatorname{Expon}(x, \mu)=\mu e^{-\mu x}$ & $P(k)=\operatorname{Expon}(k, \tau)$ & exponential law \\
$\operatorname{Normal}(x, \mu, \sigma)=\frac{1}{\sigma \sqrt{2 \pi}} e^{-\frac{(x-\mu)^{2}}{2 \sigma^{2}}}$ & $P(k)=\operatorname{Normal}(k, 1, \tau)$ & normal law \\
$\operatorname{Gamma}(x, k, \theta)=\frac{1}{\Gamma(k) \theta^{k}} x^{k-1} e^{-\frac{x}{\theta}}$ & $P(k)=\operatorname{Gamma}\left(k, \tau, e^{\tau}\right)$ & gamma law
\end{tabular}
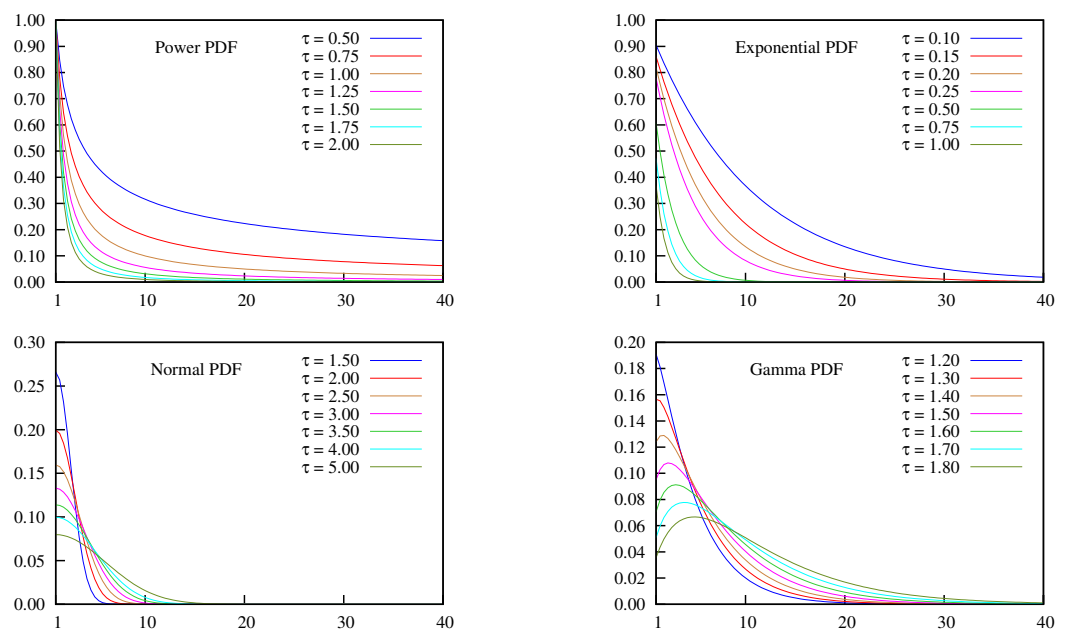

Fig. 2: Different probability distribution functions for EO 
The EO algorithm behaves very differently, depending on the PDF and the chosen value for $\tau$. Figure 2 shows the curves associated with these PDFs for a size $n=40$, picking different values for $\tau$. Clearly, with the power law, the first ranked variable has a very high probability to be selected, the probability then decreases very fast but the variables with a high rank (i.e. "good" variables) are very likely to mutate (e.g. when $\tau=0.5$ ). This results in less intensification, and suits some problems perfectly. If this behavior is unwanted, a larger value for $\tau$ may be used, nevertheless, this rapidly puts too strong a pressure on the besk ranked variables which, for some problems, will be selected too frequently. It can be difficult to find a good trade-off. On the other hand, the exponential law skews probabilities a little bit more in favor of lower ranked variables. This is clear from the shapes of the PDFs (see Figure 2). The normal (Gaussian) law is also interesting because the curve decreases slowly for the very first ranks, then more rapidly and then more slowly again: the first ranked "worst" variables will then be selected with a high but comparable priority. Finally, we found the gamma law interesting because it is not strictly decreasing and can, for instance, give more priority to the second or third variable than to the first one. With this PDF we obtained good results for tai40a with $\tau=1.5$. It is worth noticing that a shifted normal law can also be used as a non-stricly decreasing function, e.g. using $P(k)=\operatorname{Normal}\left(k, 2, e^{\tau}\right)$. It is worth noticing that the best PDF and $\tau$ combination is not the same when EO-QAP is run sequentially or in parallel.

Clearly, allowing different PDFs enhances the power of EO which can attack more problems efficiently. The user now has more precise control over the behavior of the algorithm, which remains simple with only two tunable parameters: the PDF and its $\tau$ value. It is even possible allow the user to provide his own customized PDF as a file of $n$ values $P_{1}, \ldots P_{n}$. At run-time, the prob [] array above mentionned is populated as follows: prob $[\mathrm{k}]=\frac{P_{k}}{\sum_{i=1}^{n} P_{i}}$ (each value being divided by the sum of all values to ensure the whole $\mathrm{PDF}=1$ ).

\section{Experimental Evaluation}

In this section we present experimental results on the entire QAPLIB test set. To do this, we developed an X10 implementation of EO-QAP ${ }^{3}$ Because EO is a stochastic procedure, we ran each problem 10 times and averaged the results. The number of possible experiments is very high: with different PDFs and $\tau$ values, varying the timeout, testing sequential or parallel runs, with different topologies and communication strategies, etc. We thus adopted a 3-stage protocol:

1. Attempt to solve all QAPLIB problems (134 instances) with a basic version of EO-QAP (i.e. without any tuning) and a very short timeout (in order to be able to try 10 runs for each of the 134 instances). All problems for which the Best Known Solution (BKS) was reached for every execution are definitively classified as solved. The others (solved less than 10 times) form the test set of the next stage.

\footnotetext{
${ }^{3}$ Source code and instances are available from cri-hpc1.univ-paris1.fr/qap/
} 
2. To attack the remaining problem we ran EO-QAP (with same parameters and timeout) in independent parallel multi-walks, without communication, on 32 cores of a single machine. As previously, we collected the fully solved instances which need no longer be considered. The remaining instances form the input set for the next stage.

3. The remaining problems are the hardest ones: for these, we used a cooperative parallel version of EO-QAP on 128 cores, tuning the PDF and $\tau$ value, and using a larger timeout of 10 minutes.

\subsection{Stage 1: Sequential Execution}

In this first stage, the input test set consists of the 134 QAPLIB instances, which are run sequentially on an AMD Opteron 6376 clocked at $2.3 \mathrm{GHz}$, using a single core. This is the basic version of EO-QAP with the original power-law PDF and default value for $\tau$ (see Section 2.2). For each problem we report the BKS (which is sometimes the optimum), the number of times the BKS is reached (\#BKS), i.e. the number of times the problem is solved, the average execution time (in seconds) and the the Average Percentage Deviation (APD) which is the average

of the 10 relative deviation percentages computed as follows: $100 \frac{F(s o l)-B K S}{B K S}$. We use a short timeout of 5 minutes. Even if the solver stops as soon as the BKS is reached a limited timeout is needed to be able to run the 134 instances 10 times.

Table 1 presents the whole results. Surprisingly, even with this straightforward and suboptimal setting, EO-QAP performs quite well: more than $50 \%$ of the instances get totally solved. More precisely: 68 instances are fully solved (in green), and among the remaining 66, only 25 are never solved. On average, the 41 others are solved 4.6 times (in orange). The average APD for the 66 instances not fully solved is about $2.2 \%$.

\subsection{Stage 2: Independent Parallelism}

In this stage, we ran the EO-QAP algorithm in parallel without communication with the same settings as in the first stage (default PDF, default $\tau$, timeout 5 min). The machine was the same: a quad AMD Opteron 6376 clocked at 2.3 $\mathrm{GHz}$, but using 32 cores. These parameters make it possible to assess what improvement we can easily obtain by means of parallel execution. Indeed, this from of parallelism (sometimes called embarrassingly parallelism) works by performing multiple independent walks to explore the search space. Each worker blindly explores a region of the search space, looking for a solution. The process ends as soon as any solver reaches a solution. Since all EO solvers start from a random point, we can expect that they will all visit different regions of the search space (i.e. ensuring a form of diversification), thus increasing the chance to find a solution. Such a parallelization of an algorithm is easy to implement and often behaves very well (see Section 2.3).

The results of this experiment are summarized in Table 2. This form of parallelism brings a significant improvement in performance and reach. Exactly 


\begin{tabular}{|c|c|c|c|c|c|c|c|c|c|}
\hline Problem & BKS & APD & \#BKS & time(s) & Problem & BKS & APD & \#BKS & time(s) \\
\hline bur26a & 5426670 & 0.034 & 6 & 122.817 & nug14 & 1014 & 0.000 & 10 & 0.341 \\
\hline bur $26 \mathrm{~b}$ & 3817852 & 0.101 & 5 & 151.415 & nug15 & 1150 & 0.000 & 10 & 0.293 \\
\hline bur $26 c$ & 5426795 & 0.126 & 5 & 161.905 & nug16a & 1610 & 0.373 & 5 & 150.025 \\
\hline bur26d & 3821225 & 0.120 & 2 & 251.497 & nug $16 \mathrm{~b}$ & 1240 & 0.000 & 10 & 0.008 \\
\hline bur $26 e$ & 5386879 & 0.072 & 7 & 90.776 & nug 17 & 1732 & 0.000 & 10 & 17.504 \\
\hline bur26f & 3782044 & 0.142 & 6 & 120.325 & nug18 & 1930 & 0.000 & 10 & 1.287 \\
\hline bur26g & 10117172 & 0.202 & 5 & 150.330 & nug20 & 2570 & 0.000 & 10 & 0.482 \\
\hline bur26h & 7098658 & 0.245 & 6 & 120.161 & nug21 & 2438 & 0.000 & 10 & 28.066 \\
\hline $\operatorname{chr} 12 \mathrm{a}$ & 9552 & 0.000 & 10 & 0.011 & nug22 & 3596 & 0.501 & 5 & 158.960 \\
\hline $\operatorname{chr} 12 \mathrm{~b}$ & 9742 & 0.000 & 10 & 0.005 & nug24 & 3488 & 0.034 & 9 & 87.571 \\
\hline $\operatorname{chr} 12 \mathrm{c}$ & 11156 & 0.000 & 10 & 0.178 & nug25 & 3744 & 0.000 & 10 & 0.591 \\
\hline $\operatorname{chr} 15 a$ & 9896 & 0.000 & 10 & 2.206 & nug27 & 5234 & 0.474 & 6 & 130.763 \\
\hline $\operatorname{chr} 15 b$ & 7990 & 0.000 & 10 & 0.143 & nug28 & 5166 & 0.031 & 9 & 101.182 \\
\hline $\operatorname{chr} 15 c$ & 9504 & 0.000 & 10 & 1.043 & nug30 & 6124 & 0.157 & 6 & 122.573 \\
\hline $\operatorname{chr} 18 \mathrm{a}$ & 11098 & 0.000 & 10 & 1.400 & rou12 & 235528 & 0.000 & 10 & 0.013 \\
\hline $\operatorname{chr} 18 \mathrm{~b}$ & 1534 & 0.000 & 10 & 0.041 & rou15 & 354210 & 0.000 & 10 & 0.035 \\
\hline chr20a & 2192 & 0.000 & 10 & 2.951 & rou20 & 725522 & 0.000 & 10 & 1.668 \\
\hline $\mathrm{chr} 20 \mathrm{~b}$ & 2298 & 0.000 & 10 & 3.568 & $\operatorname{scr} 12$ & 31410 & 0.000 & 10 & 0.006 \\
\hline $\operatorname{chr} 20 \mathrm{c}$ & 14142 & 0.000 & 10 & 0.632 & scr 15 & 51140 & 0.000 & 10 & 0.023 \\
\hline $\operatorname{chr} 22 \mathrm{a}$ & 6156 & 0.000 & 10 & 2.234 & scr 20 & 110030 & 0.000 & 10 & 0.334 \\
\hline $\operatorname{chr} 22 \mathrm{~b}$ & 6194 & 0.000 & 10 & 2.784 & sko42 & 15812 & 0.197 & 3 & 221.158 \\
\hline $\operatorname{chr} 25 a$ & 3796 & 0.000 & 10 & 6.803 & sko49 & 23386 & 0.073 & 2 & 250.514 \\
\hline els19 & 17212548 & 20.902 & 2 & 240.000 & sko56 & 34458 & 0.172 & 3 & 236.856 \\
\hline esc16a & 68 & 0.000 & 10 & 0.000 & sko64 & 48498 & 0.302 & 1 & 277.832 \\
\hline esc16b & 292 & 0.000 & 10 & 0.000 & sko72 & 66256 & 0.503 & 1 & 287.034 \\
\hline esc16c & 160 & 0.000 & 10 & 0.000 & sko81 & 90998 & 0.449 & 0 & 300.000 \\
\hline esc16d & 16 & 0.000 & 10 & 0.000 & sko90 & 115534 & 0.675 & 0 & 300.000 \\
\hline esc16e & 28 & 0.000 & 10 & 0.000 & sko100a & 152002 & 0.612 & 0 & 300.000 \\
\hline esc16f & 0 & 0.000 & 10 & 0.000 & sko100b & 153890 & 0.264 & 0 & 300.000 \\
\hline esc $16 \mathrm{~g}$ & 26 & 0.000 & 10 & 0.000 & sko100c & 147862 & 0.760 & 0 & 300.000 \\
\hline esc16h & 996 & 0.000 & 10 & 0.000 & sko100d & 149576 & 0.583 & 0 & 300.000 \\
\hline esc16i & 14 & 0.000 & 10 & 0.000 & sko100e & 149150 & 0.687 & 0 & 300.000 \\
\hline esc16j & 8 & 0.000 & 10 & 0.000 & sko100f & 149036 & 0.652 & 0 & 300.000 \\
\hline esc $32 a$ & 130 & 0.000 & 10 & 0.292 & ste $36 a$ & 9526 & 0.426 & 7 & 139.233 \\
\hline esc $32 b$ & 168 & 0.000 & 10 & 0.051 & ste $36 \mathrm{~b}$ & 15852 & 2.976 & 2 & 253.286 \\
\hline esc $32 c$ & 642 & 0.000 & 10 & 0.001 & ste $36 c$ & 8239110 & 0.426 & 2 & 264.393 \\
\hline esc32d & 200 & 0.000 & 10 & 6.634 & tai12a & 224416 & 0.000 & 10 & 0.011 \\
\hline esc $32 e$ & 2 & 0.000 & 10 & 0.000 & tai15a & 388214 & 0.000 & 10 & 0.089 \\
\hline esc32g & 6 & 0.000 & 10 & 0.000 & tai $17 a$ & 491812 & 0.000 & 10 & 0.292 \\
\hline esc $32 \mathrm{~h}$ & 438 & 0.000 & 10 & 18.223 & tai20a & 703482 & 0.000 & 10 & 2.637 \\
\hline esc64a & 116 & 0.000 & 10 & 0.009 & tai25a & 1167256 & 0.000 & 10 & 6.330 \\
\hline esc128 & 64 & 0.625 & 8 & 60.175 & tai30a & 1818146 & 0.000 & 10 & 9.589 \\
\hline had12 & 1652 & 0.194 & 6 & 120.000 & tai35a & 2422002 & 0.000 & 10 & 42.399 \\
\hline had14 & 2724 & 0.220 & 7 & 90.000 & tai40a & 3139370 & 0.215 & 0 & 300.000 \\
\hline had16 & 3720 & 0.032 & 4 & 180.000 & tai50a & 4938796 & 0.511 & 0 & 300.000 \\
\hline had18 & 5358 & 0.134 & 4 & 180.033 & tai60a & 7205962 & 0.537 & 0 & 300.000 \\
\hline had20 & 6922 & 0.150 & 6 & 122.549 & tai80a & 13499184 & 0.750 & 0 & 300.000 \\
\hline kra30a & 88900 & 0.983 & 4 & 180.439 & tai100a & 21052466 & 0.579 & 0 & 300.000 \\
\hline kra30b & 91420 & 0.213 & 5 & 189.252 & tai12b & 39464925 & 7.995 & 2 & 240.000 \\
\hline kra32 & 88700 & 0.826 & 5 & 150.539 & tai15b & 51765268 & 0.071 & 5 & 151.353 \\
\hline lipa20a & 3683 & 0.000 & 10 & 0.069 & tai20b & 122455319 & 21.993 & 1 & 270.000 \\
\hline lipa20b & 27076 & 0.000 & 10 & 0.005 & tai25b & 344355646 & 12.805 & 1 & 270.207 \\
\hline lipa30a & 13178 & 0.000 & 10 & 0.742 & tai30b & 637117113 & 15.479 & 1 & 270.022 \\
\hline lipa30b & 151426 & 0.000 & 10 & 0.037 & tai35b & 283315445 & 7.703 & 0 & 300.000 \\
\hline lipa40a & 31538 & 0.000 & 10 & 1.962 & tai40b & 637250948 & 10.085 & 0 & 300.000 \\
\hline lipa 40b & 476581 & 0.000 & 10 & 0.067 & tai50b & 458821517 & 6.803 & 0 & 300.000 \\
\hline lipa50a & 62093 & 0.000 & 10 & 4.245 & tai60b & 608215054 & 6.559 & 0 & 300.000 \\
\hline lipa50b & 1210244 & 0.000 & 10 & 0.113 & tai80b & 818415043 & 5.402 & 0 & 300.000 \\
\hline lipa60a & 107218 & 0.000 & 10 & 18.825 & tai $100 \mathrm{~b}$ & 1185996137 & 4.750 & 0 & 300.000 \\
\hline lipa60b & 2520135 & 0.000 & 10 & 2.282 & tai150b & 498896643 & 2.686 & 0 & 300.000 \\
\hline lipa70a & 169755 & 0.000 & 10 & 57.737 & tai64c & 1855928 & 0.450 & 0 & 300.000 \\
\hline lipa70b & 4603200 & 0.000 & 10 & 8.288 & tai256c & 44759294 & 0.431 & 0 & 300.000 \\
\hline lipa80a & 253195 & 0.047 & 9 & 158.337 & tho 30 & 149936 & 0.184 & 9 & 87.784 \\
\hline lipa80b & 7763962 & 0.000 & 10 & 18.203 & tho 40 & 240516 & 0.147 & 2 & 241.778 \\
\hline lipa90a & 360630 & 0.221 & 5 & 256.062 & tho150 & 8133398 & 1.196 & 0 & 300.000 \\
\hline lipa90b & 12490441 & 0.000 & 10 & 20.193 & wil50 & 48816 & 0.153 & 0 & 300.000 \\
\hline nug12 & 578 & 0.000 & 10 & 0.012 & will100 & 273038 & 0.409 & 0 & 300.000 \\
\hline
\end{tabular}

Table 1: Sequential execution (power-law, default $\tau$, timeout $=300 \mathrm{~s}$ ) 


\begin{tabular}{|c|c|c|c|c|}
\hline Problem & BKS & APD & \#BKS & time(s) \\
\hline bur26a & 5426670 & 0.000 & 10 & 0.027 \\
\hline bur26b & 3817852 & 0.000 & 10 & 0.021 \\
\hline bur26c & 5426795 & 0.000 & 10 & 0.009 \\
\hline bur26d & 3821225 & 0.000 & 10 & 9.311 \\
\hline bur $26 e$ & 5386879 & 0.000 & 10 & 0.010 \\
\hline bur26f & 3782044 & 0.000 & 10 & 0.009 \\
\hline bur $26 \mathrm{~g}$ & 10117172 & 0.000 & 10 & 0.006 \\
\hline bur $26 \mathrm{~h}$ & 7098658 & 0.000 & 10 & 0.010 \\
\hline els19 & 17212548 & 0.421 & 9 & 30.007 \\
\hline esc128 & 64 & 0.000 & 10 & 0.036 \\
\hline had12 & 1652 & 0.000 & 10 & 0.000 \\
\hline had14 & 2724 & 0.000 & 10 & 0.000 \\
\hline had16 & 3720 & 0.000 & 10 & 0.000 \\
\hline had18 & 5358 & 0.000 & 10 & 0.001 \\
\hline had20 & 6922 & 0.000 & 10 & 0.001 \\
\hline kra30a & 88900 & 0.134 & 9 & 30.544 \\
\hline kra30b & 91420 & 0.000 & 10 & 2.485 \\
\hline kra32 & 88700 & 0.000 & 10 & 0.195 \\
\hline lipa80a & 253195 & 0.000 & 10 & 15.001 \\
\hline lipa90a & 360630 & 0.000 & 10 & 32.361 \\
\hline nug16a & 1610 & 0.000 & 10 & 0.001 \\
\hline nug22 & 3596 & 0.000 & 10 & 0.002 \\
\hline nug24 & 3488 & 0.000 & 10 & 0.004 \\
\hline nug27 & 5234 & 0.000 & 10 & 0.006 \\
\hline nug28 & 5166 & 0.000 & 10 & 0.059 \\
\hline nug30 & 6124 & 0.000 & 10 & 0.268 \\
\hline sko42 & 15812 & 0.000 & 10 & 1.138 \\
\hline sko49 & 23386 & 0.000 & 10 & 40.118 \\
\hline sko56 & 34458 & 0.001 & 9 & 71.411 \\
\hline sko64 & 48498 & 0.005 & 8 & 109.435 \\
\hline sko72 & 66256 & 0.041 & 3 & 236.308 \\
\hline sko81 & 90998 & 0.044 & 1 & 271.685 \\
\hline sko90 & 115534 & 0.125 & 1 & 288.550 \\
\hline
\end{tabular}

\begin{tabular}{|c|c|c|c|c|}
\hline Problem & BKS & APD & \#BKS & time(s) \\
\hline sko100a & 152002 & 0.117 & 0 & 300.000 \\
\hline sko100b & 153890 & 0.112 & 0 & 300.000 \\
\hline sko100c & 147862 & 0.056 & 0 & 300.000 \\
\hline sko100d & 149576 & 0.133 & 0 & 300.000 \\
\hline sko100e & 149150 & 0.059 & 0 & 300.000 \\
\hline sko100f & 149036 & 0.144 & 0 & 300.000 \\
\hline ste36a & 9526 & 0.000 & 10 & 1.148 \\
\hline ste $36 \mathrm{~b}$ & 15852 & 0.000 & 10 & 2.035 \\
\hline ste $36 \mathrm{c}$ & 8239110 & 0.000 & 10 & 5.148 \\
\hline tai40a & 3139370 & 0.074 & 0 & 300.000 \\
\hline tai50a & 4938796 & 0.286 & 0 & 300.000 \\
\hline tai60a & 7205962 & 0.302 & 0 & 300.000 \\
\hline tai80a & 13499184 & 0.497 & 0 & 300.000 \\
\hline tai100a & 21052466 & 0.419 & 0 & 300.000 \\
\hline tai12b & 39464925 & 0.000 & 10 & 0.001 \\
\hline tai15b & 51765268 & 0.000 & 10 & 0.001 \\
\hline tai20b & 122455319 & 0.045 & 9 & 30.003 \\
\hline tai25b & 344355646 & 0.074 & 8 & 94.831 \\
\hline tai30b & 637117113 & 0.638 & 6 & 122.746 \\
\hline tai35b & 283315445 & 0.364 & 3 & 229.160 \\
\hline tai40b & 637250948 & 0.339 & 7 & 91.070 \\
\hline tai50b & 458821517 & 1.222 & 0 & 300.000 \\
\hline tai60b & 608215054 & 1.318 & 0 & 300.000 \\
\hline tai80b & 818415043 & 2.012 & 0 & 300.000 \\
\hline tai100b & 1185996137 & 0.900 & 0 & 300.000 \\
\hline tai150b & 498896643 & 1.546 & 0 & 300.000 \\
\hline tai64c & 1855928 & 0.012 & 8 & 60.004 \\
\hline tai256c & 44759294 & 0.294 & 0 & 300.000 \\
\hline tho30 & 149936 & 0.000 & 10 & 0.235 \\
\hline tho40 & 240516 & 0.002 & 8 & 77.261 \\
\hline tho150 & 8133398 & 0.436 & 0 & 300.000 \\
\hline wil50 & 48816 & 0.000 & 10 & 27.545 \\
\hline wil100 & 273038 & 0.111 & 0 & 300.000 \\
\hline
\end{tabular}

Table 2: Independent parallelism on 32 cores (timeout $=300 \mathrm{~s}$ )

half of the 66 problem instances now become fully solved (the average time being $3.2 s$ ). However, among the 33 remaining ones, 19 remain never solved and, on average, the remaining 14 get solved 6 out of 10 times. Moreover, the average APD over the not fully solved 33 is $0.372 \%$. This contrasts with the previous situation $(2.2 \%)$, which indicates a significant improvement in the quality of solutions: even when the optimum is not reached, the solution which was found is close.

\subsection{Stage 3: Cooperative Parallelism}

In this final experiment, we attacked the 33 hardest instances with parallelism and cooperation. This was simplified thanks to the CPLS framework which provides the necessary abstraction layers and already handles the communication (see Section 2.3). The sequential EO-QAP needed a very simple adaptation: every $R$ iterations it has to send its current configuration to the Elite Pool and, every $U$ iterations, it retrieves a configuration from the pool, which it nondeterministically adopts it if it is better than the current one. The CPLS system

\footnotetext{
${ }^{4}$ With a probability $p$ Adopt.
} 
already provides library functions for all these operations. The resulting solver is then composed of several EO-QAP instances running in parallel, which cooperate by communicating in order to converge faster on a solution. As per [7, the CPLS parameters which control the cooperation are as follows:

- Team Size (NPT): we tested various configurations and defined NPT $=16$. There are thus 8 teams composed of 16 explorer nodes each running the EO-QAP procedure. This is constant for all problems.

- Report Interval $(R)$ : we manually tuned it (starting from the average number of iterations collected during the previous stage divided by 10).

- Update Interval $(U)$ : we experimented different ratio and retained $U / R=2$.

- Elite Pool (EP): its size is fixed to 4 for all problems.

- pAdopt: is set to 1. An EO-QAP instance receiving a better configuration than its current one always switches to this new one.

This experiment has been carried out on a cluster of 16 machines, each with $4 \times 16$-core AMD Opteron $6376 \mathrm{CPUs}$ running at $2.3 \mathrm{GHz}$ and $128 \mathrm{~GB}$ of RAM. The nodes are interconnected with InfiniBand FDR $4 \times$ (i.e. 56 GBPS.) We had access to 4 nodes and used up to 32 cores per node, i.e. 128 cores. We stay with a timeout of 5 minutes. Finally, we tested deeply two PDFs (power and exponential) and their $\tau$ value and retained the best combination for each problem instance (we could not yet test the Normal law and the Gamma law, while efficient in sequential seems, not well suited for parallelism or else using a different $\tau$ value).

Table 3 presents the results obtained on the hardest instances. The table also reports the average number of iterations, the report and update interval ( $R$ and $U)$, the number of times the winning algorithm has adopted an elite configuration, the PDF and $\tau$ value used. In this last stage, 15 new problems become fully solved. Moreover the average time to solve them is only 24.5 seconds. Only 8 remain unsolved. On average, the remaining 10 get solved 5 out of 10 times. Moreover, the average APD over the 18 not fully solved is only $0.25 \%$. Even when the optimum is not reached the returned solution is close to this optimum.

The table shows that an efficient execution corresponds to a limited number of "adoptions" of an elite configuration (less than 5 changes). This is directly correlated to the value of $R$ and $U$. There are some exceptions like tai25b and tho40 which are both fully solved. We plan to analyze in details these both situations (e.g. varying $\mathrm{R}$ and $\mathrm{U}$ ).

Regarding the power and exponential PDF, there is no winner. It is worth noticing that sometimes the difference is huge. For instance, sko90 is solved 9 times with the power law but only 2 times with the exponential law. The reverse occurs for tai $X X$ a for which the exponential law performs much better.

The performance of the cooperative parallel EO-QAP, is on par with the best competing solutions, while retaining a much simpler internal structure 32]. Due to space limitations, we do not develop this further. 


\begin{tabular}{|c|c|c|c|c|c|c|c|c|c|c|}
\hline Problem & BKS & APD & \#BKS & time(s) & \#iters & $R$ & $U$ & \#adopt & PDF & $\tau$ \\
\hline els19 & 17212548 & 0.000 & 10 & 0.003 & 23 & 10 & 20 & 0.2 & pow & 1.70 \\
\hline kra30a & 88900 & 0.000 & 10 & 0.026 & 599 & 100 & 200 & 2.6 & pow & 0.20 \\
\hline sko56 & 34458 & 0.000 & 10 & 4.776 & 35658 & 7000 & 14000 & 2.5 & pow & 0.60 \\
\hline sko64 & 48498 & 0.000 & 10 & 4.822 & 26494 & 7000 & 14000 & 1.5 & pow & 0.60 \\
\hline sko72 & 66256 & 0.000 & 10 & 13.442 & 57956 & 15000 & 30000 & 1.4 & pow & 0.80 \\
\hline sko81 & 90998 & 0.008 & 7 & 118.905 & 399748 & 20000 & 40000 & 9.4 & pow & 1.00 \\
\hline sko90 & 115534 & 0.000 & 10 & 92.951 & 253547 & 25000 & 50000 & 5.0 & pow & 0.60 \\
\hline sko100a & 152002 & 0.012 & 5 & 224.206 & 492441 & 50000 & 100000 & 4.2 & pow & 1.00 \\
\hline sko100b & 153890 & 0.001 & 8 & 146.679 & 322560 & 50000 & 100000 & 2.6 & pow & 1.00 \\
\hline sko100c & 147862 & 0.000 & 10 & 145.032 & 319871 & 50000 & 100000 & 2.4 & pow & 1.00 \\
\hline sko100d & 149576 & 0.014 & 6 & 200.948 & 442626 & 50000 & 100000 & 3.6 & pow & 1.00 \\
\hline sko100e & 149150 & 0.000 & 10 & 103.370 & 228094 & 50000 & 100000 & 1.6 & pow & 1.00 \\
\hline sko100f & 149036 & 0.011 & 4 & 245.929 & 542031 & 50000 & 100000 & 4.8 & pow & 1.00 \\
\hline tai40a & 3139370 & 0.022 & 7 & 171.113 & 2701406 & 350000 & 700000 & 3.4 & $\exp$ & 0.18 \\
\hline tai50a & 4938796 & 0.026 & 5 & 208.882 & 1997696 & 350000 & 700000 & 2.4 & $\exp$ & 0.16 \\
\hline tai60a & 7205962 & 0.132 & 2 & 285.329 & 1833023 & 400000 & 800000 & 1.9 & $\exp$ & 0.17 \\
\hline tai80a & 13499184 & 0.385 & 0 & 300.000 & 1037282 & 400000 & 800000 & 1.0 & $\exp$ & 0.16 \\
\hline tai100a & 21052466 & 0.297 & 0 & 300.000 & 657489 & 100000 & 200000 & 3.0 & exp & 0.13 \\
\hline tai20b & 122455319 & 0.000 & 10 & 0.001 & 69 & 20 & 40 & 0.8 & $\exp$ & 0.38 \\
\hline tai25b & 344355646 & 0.000 & 10 & 0.600 & 23331 & 400 & 800 & 17.0 & pow & 1.40 \\
\hline tai30b & 637117113 & 0.000 & 10 & 0.121 & 3360 & 500 & 1000 & 3.0 & pow & 0.20 \\
\hline tai35b & 283315445 & 0.000 & 10 & 0.737 & 15659 & 500 & 1000 & 14.2 & pow & 0.80 \\
\hline tai40b & 637250948 & 0.000 & 10 & 0.061 & 973 & 500 & 1000 & 0.4 & $\exp$ & 0.12 \\
\hline tai50b & 458821517 & 0.214 & 2 & 266.446 & 2571166 & 250000 & 500000 & 4.5 & $\exp$ & 0.03 \\
\hline tai60b & 608215054 & 0.205 & 3 & 256.836 & 1676681 & 250000 & 500000 & 2.6 & pow & 0.40 \\
\hline tai80b & 818415043 & 1.192 & 0 & 300.000 & 1035635 & 45000 & 90000 & 8.8 & pow & 1.05 \\
\hline tai100b & 1185996137 & 0.465 & 0 & 300.000 & 658761 & 50000 & 100000 & 5.5 & pow & 1.02 \\
\hline tai150b & 498896643 & 1.088 & 0 & 300.000 & 281965 & 70000 & 140000 & 1.5 & pow & 0.94 \\
\hline tai64c & 1855928 & 0.000 & 10 & 0.010 & 27 & 6 & 12 & 0.3 & $\exp$ & 0.04 \\
\hline tai256c & 44759294 & 0.263 & 0 & 300.000 & 81624 & 10000 & 20000 & 1.3 & exp & 0.04 \\
\hline tho 40 & 240516 & 0.000 & 10 & 1.243 & 20091 & 22000 & 44000 & 0.2 & pow & 1.00 \\
\hline tho150 & 8133398 & 0.144 & 0 & 300.000 & 281711 & 50000 & 100000 & 1.7 & pow & 1.00 \\
\hline wil100 & 273038 & 0.061 & 0 & 300.000 & 662233 & 50000 & 100000 & 5.4 & $\exp$ & 0.09 \\
\hline
\end{tabular}

Table 3: Cooperative parallelism on 128 cores $($ timeout $=300 \mathrm{~s})$

\section{Conclusion and Further Work}

We have proposed EO-QAP: an Extremal Optimization (EO) procedure for QAP. The basic sequential version of EO-QAP, while simple behaves rather well on several instances of QAPLIB. To attack hardest instances we first proposed a simple extension to the original EO procedure allowing for different probability distribution functions (PDF). The user can select the most adequate PDF depending on the degree of intensification wanted. Moreover, we resorted to cooperative parallelism using a framework written in the X10 parallel language, which provides the user with a fine degree of control of the intensification and diversification for the search. The cooperative version of EO-QAP displays very good results: using 128 cores, 116 instances of QAPLIB are systematically solved at each execution, 10 instances are solved half the time and only 8 instances remain unsolved (but the obtained solution is near to the optimum).

We now plan to attack other known hard instances. Future work includes the study of a default parameter for the other PDFs (e.g. exponential) and how to take into account the hardness of the problem to define this value (e.g. in terms of the landscape ruggedness of the instance to solve). Moreover, we plan 
to explore portfolio approaches, i.e. ones which combine multiple solvers as well as experiment with techniques for parameter auto-tuning. This experimentation entails a deep analysis of the parallel performance behavior.

\section{Acknowledgments}

The authors wish to acknowledge Stefan Boettcher (Emory University) for his explanations about the Extremal Optimization method. The experimentation used the cluster of the University of Évora, which was partly funded by grants ALENT-07-0262-FEDER-001872 and ALENT-07-0262-FEDER-001876.

\section{References}

1. Koopmans, T.C., Beckmann, M.: Assignment Problems and the Location of Economic Activities. Econometrica 25(1) (1957) 53-76

2. Commander, C.W.: A survey of the quadratic assignment problem, with applications. Morehead Electronic Journal of Applicable Mathematics 4 (2005) MATH2005-01

3. Bhati, R.K., Rasool, A.: Quadratic Assignment Problem and its Relevance to the Real World: A Survey. International Journal of Computer Applications 96(9) (2014) 42-47

4. Sahni, S., Gonzalez, T.: P-Complete Approximation Problems. Journal of the ACM 23(3) (1976) 555-565

5. Boettcher, S., Percus, A.: Nature's way of optimizing. Artificial Intelligence 119(12) (2000) 275-286

6. Randall, M., Lewis, A.: Intensification Strategies for Extremal Optimisation. In: Simulated Evolution and Learning - 8th International Conference, (SEAL), Kanpur, India. Volume 6457 of LNCS., Springer (2010) 115-124

7. Munera, D., Diaz, D., Abreu, S., Codognet, P.: A Parametric Framework for Cooperative Parallel Local Search. In Blum, C., Ochoa, G., eds.: European Conference on Evolutionary Computation in Combinatorial Optimisation (EvoCOP). Volume 8600 of Lecture Notes in Computer Science., Springer (2014) 13-24

8. Charles, P., Grothoff, C., Saraswat, V., Donawa, C., Kielstra, A., Ebcioglu, K., Von Praun, C., Sarkar, V.: X10: An Object-Oriented Approach to Non-Uniform Cluster Computing. In: SIGPLAN Conference on Object-oriented Programming, Systems, Languages, and Applications, San Diego, CA, USA, ACM (2005) 519-538

9. Saraswat, V., Tardieu, O., Grove, D., Cunningham, D., Takeuchi, M., Herta, B.: A Brief Introduction to X10 (for the High Performance Programmer). Technical report (2012)

10. Burkard, R.E.: Quadratic Assignment Problems. In Pardalos, P.M., Du, D.Z., Graham, R.L., eds.: Handbook of Combinatorial Optimization (2nd edition). Springer New York (2013) 2741-2814

11. Loiola, E.M., de Abreu, N.M.M., Netto, P.O.B., Hahn, P., Querido, T.M.: A survey for the quadratic assignment problem. European Journal of Operational Research 176(2) (2007) 657-690

12. Zaied, A.N.H., Shawky, L.A.E.f.: A Survey of Quadratic Assignment Problems. International Journal of Computer Applications 101(6) (2014) 28-36 
13. Said, G.A.E.N.A., Mahmoud, A.M., El-Horbaty, E.S.M.: A Comparative Study of Meta-heuristic Algorithms for Solving Quadratic Assignment Problem. International Journal of Advanced Computer Science and Applications (IJACSA) 5(1) (2014)

14. Boettcher, S., Percus, A.G.: Extremal Optimization: an Evolutionary Local-Search Algorithm. In: Computational Modeling and Problem Solving in the Networked World. Volume 21. Springer US (2003)

15. Boettcher, S.: Extremal Optimization. In Hartmann, A.K., Rieger, H., eds.: New Optimization Algorithms to Physics. Wiley-VCH Verlag, Berlin (2004) 227-251

16. Bak, P., Tang, C., Wiesenfeld, K.: Self-Organized Crtiticality: An Explenation of 1/f Noise. Physical Review Letters 59(4) (1987) 381-384

17. Bak, P.: How Nature Works: The Science of Self-organized Criticality. 1st edn. Copernicus (Springer) (1996)

18. Bak, P., Sneppen, K.: Punctuated equilibrium and criticality in a simple model of evolution. Physical Review Letters 71(24) (1993) 4083-4086

19. Boettcher, S., Percus, A.G.: Optimization with Extremal Dynamics. Physical Review Letters 86(23) (2001) 5211-5214

20. De Sousa, F.L., Ramos, F.M.: Function optimization using extremal dynamics. International Conference on Inverse Problems in Engineering Rio de Janeiro, Brazil (2002)

21. De Sousa, F.L., Vlassov, V., Ramos, F.M.: Generalized Extremal Optimization for Solving Complex Optimal Design Problems. International Conference on Genetic and Evolutionary Computation (2003) 375-376

22. Zhou, T., Bai, W.J., Cheng, L.J., Wang, B.H.: Continuous Extremal Optimization for Lennard-Jones Clusters. Physical Review E 72(1) (2005)

23. Alba, E.: Parallel Metaheuristics: A New Class of Algorithms. Wiley-Interscience (2005)

24. Alba, E., Luque, G., Nesmachnow, S.: Parallel Metaheuristics: Recent Advances and New Trends. International Transactions in Operational Research 20(1) (2013) $1-48$

25. Diaz, D., Abreu, S., Codognet, P.: Parallel Constraint-Based Local Search on the Cell/BE Multicore Architecture. In: Studies in Computational Intelligence. Volume 315. (2010) 265-274

26. Verhoeven, M., Aarts, E.: Parallel Local Search. Journal of Heuristics 1(1) (1995) $43-65$

27. Caniou, Y., Codognet, P., Richoux, F., Diaz, D., Abreu, S.: Large-scale parallelism for constraint-based local search: the costas array case study. Constraints 20(1) (2014) 1-27

28. Toulouse, M., Crainic, T., Sansó, B.: Systemic Behavior of Cooperative Search Algorithms. Parallel Computing (2004) 57-79

29. Munera, D., Diaz, D., Abreu, S., Codognet, P.: Flexible Cooperation in Parallel Local Search. In: Symposium on Applied Computing (SAC), New York, New York, USA, ACM Press (2014) 1360-1361

30. Minton, S., Philips, A., Johnston, M.D., Laird, P.: Minimizing Conflicts: A Heuristic Repair Method for Constraint-Satisfaction and Scheduling Problems. Journal of Artificial Intelligence Research 58 (1993) 161-205

31. Taillard, É.D.: Comparison of iterative searches for the quadratic assignment problem. Location Science 3(2) (1995) 87-105

32. James, T., Rego, C., Glover, F.: A Cooperative Parallel Tabu Search Algorithm for the Quadratic Assignment Problem. European Journal of Operational Research (2009) 